\title{
$\beta$-Glucuronidase Deficiency in a Dog: a Model of Human Mucopolysaccharidosis VII
}

\author{
MARK E. HASKINS, ROBERT J. DESNICK, NICOLA DIFERRANTE, PETER F. JEZYK, AND \\ DONALD F. PATTERSON \\ Sections of Pathology [M.E.H.] and Medical Genetics [M.E.H., P.F.J., D.F.P.], University of Pennsylvania, \\ School of Veterinary Medicine and the University of Pennsylvania Genetics Center, \\ Philadelphia, Pennsylvania 19104, Marrs McLean Department of Biochemistry, Baylor College of Medicine, \\ Houston, Texas 77030 [N.D.], Division of Medical Genetics, Mt. Sinai School of Medicine, \\ New York, New York 10029 [R.J.D.]
}

\begin{abstract}
Summary
This report describes a third mucopolysaccharidosis in animals: canine mucopolysaccharidosis VII. The affected dog was the offspring of a father-daughter mating. Weakness in the rear legs was evident at 8 weeks of age and became progressively worse. He had a large head, a shortened maxilla, and corneal granularities. Most joints were extremely lax, easily subluxated, with joint capsules that were swollen and fluctuant.

The dog was alert and had apparently normal pain perception. At 13 months of age, there was radiographic evidence of extensive skeletal disease including bilateral femoral head luxation, abnormalities in the shape and density of the carpal and tarsal bones, radiolucent lesions of the epiphyseal regions of most long bones, and cervical vertebral dysplasia and platyspondylia. The electrophoretic pattern of precipitated glycosaminoglycans indicated a predominance of chondroitin sulfate.

The animal died suddenly from gastric dilatation. There was generalized hepatomegaly, thickening of the atrioventricular heart valves, and generalized polyarthropathy. Vacuolated cytoplasm was observed in hepatocytes, keratocytes, fibroblasts, chondrocytes and cells of the synovial membrane, retinal pigment epithelium, and cardiac valves. Neurons had cytoplasmic vacuoles. Electron microscopy demonstrated membrane-bound cytoplasmic inclusions in polymorphonuclear leukocytes, hepatocytes, synovium, heart valves and spleen.

The activities of 12 lysosomal hydrolases were determined in liver from the affected and control dogs: $\beta$-glucuronidase (EC 3.2.1.31), $\beta$-hexosaminidases A and B (EC 3.2.1.30), $\alpha$-hexosaminidase (EC 3.2.1.-), $\alpha$-L-iduronidase (EC 3.2.1.76), $\alpha$-galactosidase A (EC 3.2.1.22), $\beta$-galactosidase (EC 3.2.1.23), arylsulfatases $A$ and B (EC 3.1.6.1), acid $\alpha$-mannosidase (EC 3.2.1.24), acid $\beta$-mannosidase (EC 3.2.1.25), and $N$-acetyl-D-galactosamine-6-sulfate sulfatase (EC 3.1.6.-). The activity of $\beta$-glucuronidase was reduced to less than $2 \%$ of the normal mean value of normal controls.
\end{abstract}

\section{Abbreviations}

MPS, mucopolysaccharidoses

GAG, glycosaminoglycan

EM, electron microscopy

Received November 29, 1983; accepted March 24, 1984

Reprint requests may be addressed to Mark Haskins, V.M.D., Ph.D., Veterinary Pathology, 3800 Spruce Street, Philadelphia, PA 19104.

This work was supported by National Institutes of Health Grants AM 25759 , and AM 26482, the University of Pennsylvania Human Genetics Center Grant GM 20138, and the Mrs. Cheever Porter Foundation.
The MPS are a group of genetic diseases resulting from defective degradation of GAG (12). Each syndrome has a characteristic combination of clinical signs, urinary GAG excretion, and a specific lysosomal enzyme deficiency. The general clinical characteristics include dysostosis multiplex, facial dysmorphia, hepatosplenomegaly, mental retardation, lysosomal accumulation and urinary excretion of GAG, and metachromatic granules in blood leukocytes. Animal models of MPS I [domestic cat (7), plott hound dog (17)], and VI [Siamese cat (10)] have been described. This report describes a third mucopolysaccharidosis in animals: canine mucopolysaccharidosis VII.

\section{MATERIALS AND METHODS}

Four animals were studied: The affected animal (referred to us by Dr. Niels Pederson, University of California, Davis) its parents, and a newborn half-sibling of the affected animal (Fig. 1).

Urinary Glycosaminoglycans. Urine spot tests for GAG were performed (1). Urine was mixed with $10 \%$ cetylpyridinium chloride, the precipitate was treated as previously described (4), and the residue was dissolved in $2 \mathrm{ml}$ of distilled water. Aliquots were used for microelectrophoresis on a $0.7 \%$ agarose gel in $0.05 \mathrm{M}$ 1,3-diaminopropane:acetate buffer, $\mathrm{pH} 9.0$. Digestion of the crude urinary GAG with chondroitinase AC (EC 4.2.2.5) and $\mathrm{ABC}$ (EC 4.2.2.4) (Sigma Chemical Co.) was performed as previously described (14).

Pathology. Blood samples were collected in EDTA for light microscopy or in heparin for EM. Leukocytes for EM were separated by a modification of the method of Skoog and Beck (18). Leukocytes and other tissues for EM were fixed in 5\% buffered glutaraldehyde for $48 \mathrm{~h}$, embedded in Spurr's medium (20), sectioned on a Sorvall MT2-B microtome to $700 \AA$, stained with lead citrate-uranyl acetate, and examined using a Zeiss EM 9S-2 transmission electron microscope. Tissues for light microscopy were fixed in $10 \%$ buffered formalin, paraffin embedded, and sectioned. Routine stains included hematoxylin and eosin, cresyl violet, trichrome phosphotungstic acid-hematoxylin, and luxol blue-periodic acid Schiff-hematoxylin-eosin.

Enzyme assays. Liver $(5 \mathrm{~g})$ from the propositus, sire, dam, half-sibling, and 11 control dogs was thawed, cut into small pieces, and washed with cold saline to remove blood. Hepatic tissues $(0.5 \mathrm{~g})$ were homogenized in 5 volumes of $10 \mathrm{mM}$ sodium phosphate buffer, $\mathrm{pH} 6.0$, containing $0.2 \%$ Triton $\mathrm{X}-100$. The homogenates were centrifuged at $17,000 \times g$ for $30 \mathrm{~min}$ at $4^{\circ} \mathrm{C}$. The supernatants were removed and used as enzyme source for the determination of arylsulfatase A and B activities using $p$ nitrocatechol as substrate (11) and for the lysosomal glycosidases 

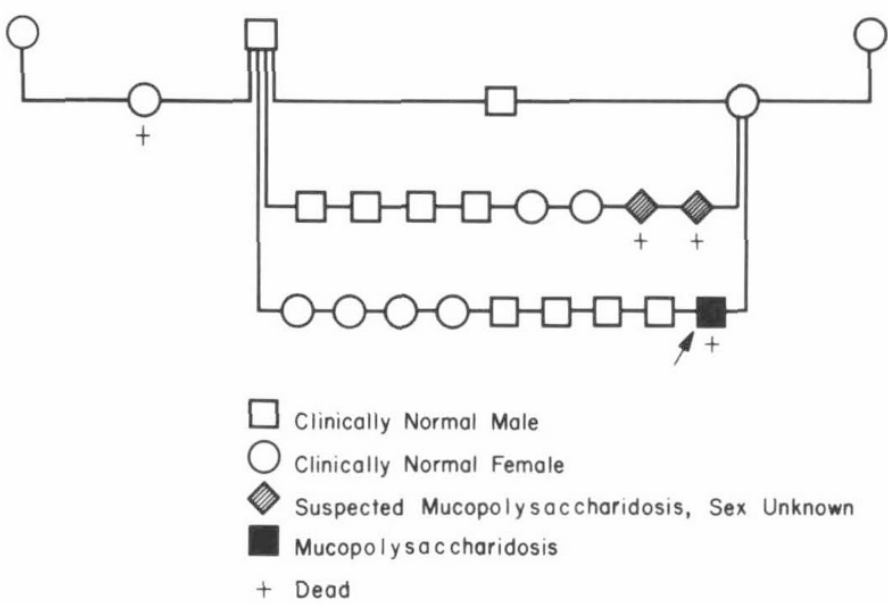

Fig. 1. The pedigree of the affected animal. Note that he was the product of a father-daughter mating.

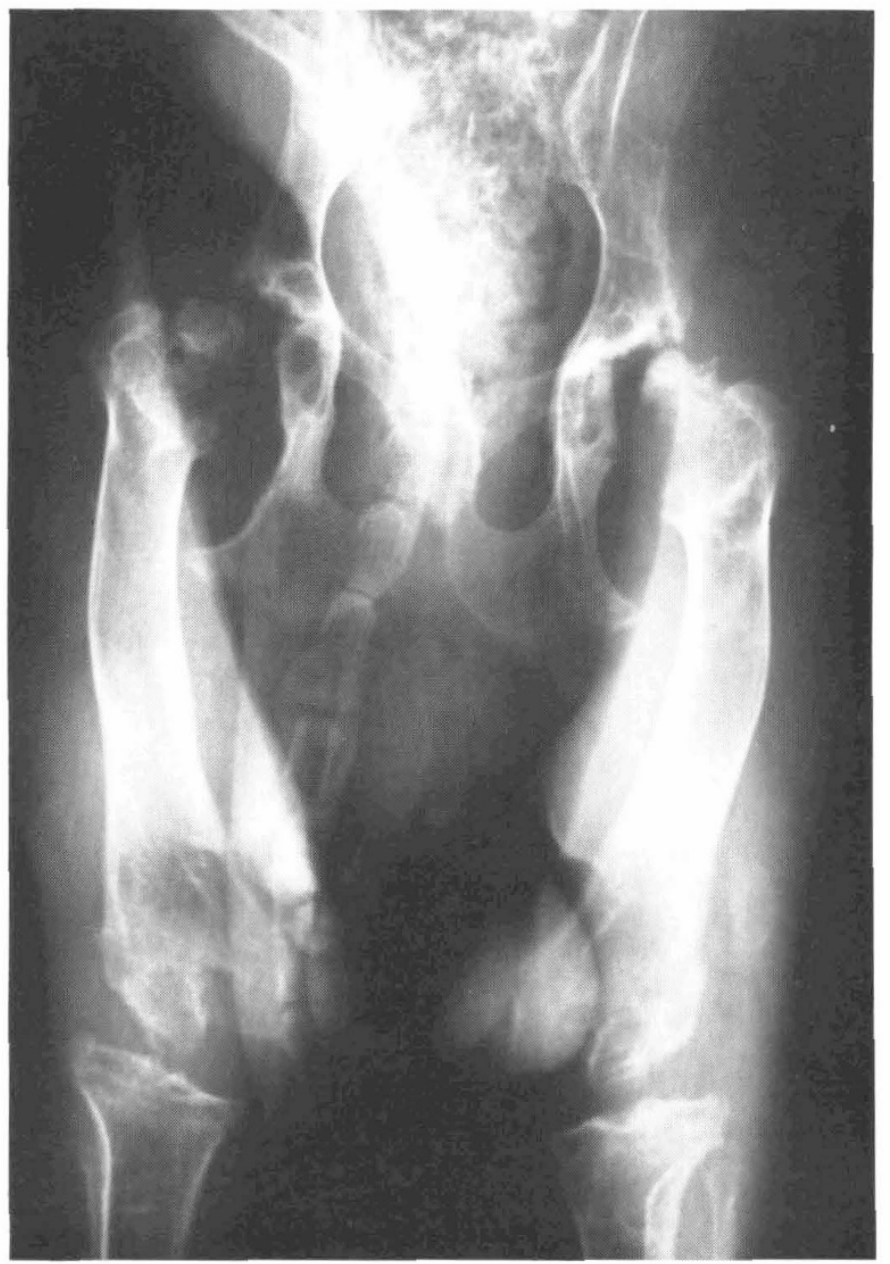

Fig. 2. Ventro-dorsal (anterior-posterior in man) radiograph of the coxofemoral joints illustrating bilateral femoral head luxation with severe irregularities in outline and increased bone density (sclerosis) of the femoral heads and acetabulae. The femoral necks are narrow and the stifle (knee) joint is at an abnormal angle with bilateral patella luxation.

using the appropriate 4-methylumbelliferyl-glycoside as substrate $[\alpha$-mannosidase A and B (3), $\beta$-glucuronidase (5), $\beta$-hexosaminidase A and B (8), $\beta$-glucosidase (16), $\alpha$-hexosaminidase (15), $\alpha$ L-iduronidase (7), $\alpha$-galactosidase A (2), and $\beta$-galactosidase (2)].

For determination of $\mathrm{N}$-acetyl-D-galactosamine-6-sulfate sulfatase activity, the hepatic tissue was homogenized in 5 volumes of $10 \mathrm{mM}$ sodium phosphate buffer, $\mathrm{pH} 6.6$, containing $0.01 \mathrm{M}$ 2 -mercaptoethanol and centrifuged. The supernatants were removed, solid ammonium sulfate was added to bring the supernatants to $65 \%$ saturation, and the mixture was stirred overnight at $4^{\circ} \mathrm{C}$. The precipitate was removed by centrifugation and used as enzyme source (21) after exhaustive dialysis against the phosphate buffer.

Clinical features. The affected male, mixed breed dog was the offspring of a father-daughter mating. Weakness in the rear legs was evident at 8 weeks of age and became progressively worse. At 8 months of age, the animal was incapable of standing or fully supporting his weight and had a large head in proportion to his body, a shortened maxilla, and a relatively long mandible. The tongue protruded beyond the labial margins and the incisors were peg-shaped and wide-spaced. Both corneas contained diffuse fine stromal granularities with small, peripheral areas of edema and focal, central cholesterol deposits. Both the axial and appendicular skeletons were deformed: the spine was shortened and the rib cage was compressed dorsoventrally (anterior-posterior in man). The long bones were shortened and curved. Most joints were extremely lax, easily subluxated and crepitant, with joint capsules that were swollen and fluctuant. Both patellas were luxated medially.

The dog was alert and had apparently normal pain perception in all areas. Function of all cranial nerves was evaluated as within normal limits; segmental reflexes appeared normal, within the limits imposed by severe skeletal disease. Many of the muscles of locomotion were atrophied. The animal had an active libido and his semen was used to artificially inseminate three females, one of which conceived. Cardiovascular examination revealed no abnormalities.

At 13 months of age the animal had radiographic evidence of extensive skeletal disease including bilateral femoral head luxation (Fig. 2), extensive abnormalities in the shape and density of the carpal and tarsal bones, radiolucent lesions of the epiphyseal regions of most long bones, and cervical vertebral dysplasia and platyspondylia. These lesions were more severe than when he was first examined at 8 months of age.
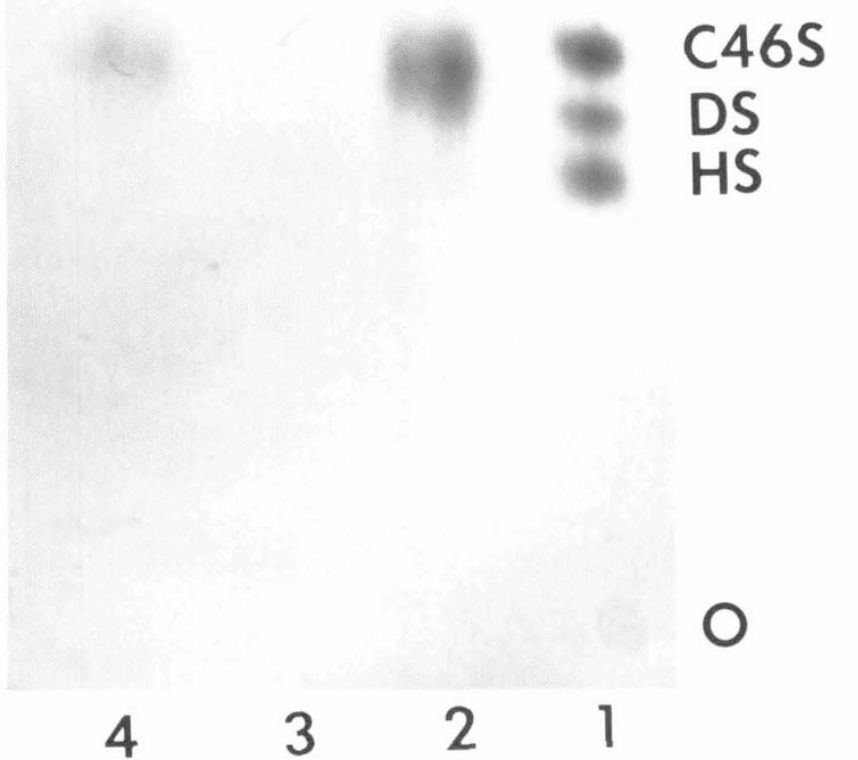

Fig. 3. The electrophoretogram of urine precipitated with cetylpyridinium chloride. Lane 1 contained standards; lane 2, urine from the affected dog; lane 3, urine digested with chondroitinase $\mathrm{ABC}$; lane 4, urine digested with chondroitinase AC. C46S, chondroitin 4 and 6 sulfates; $D S$, dermatan sulfate; $H S$, heparan sulfate; $O$, origin. 
In peripheral blood smears stained with toluidine-blue, most lymphocytes and granulocytes contained metachromatic cytoplasmic granules (Alder-Reilly bodies).

Urine. Metabolic screening tests of the urine yielded a positive spot for GAG in the affected dog and no spot for control urine. The electrophoretic pattern of precipitated GAG from the affected dog's urine indicated a predominance of chondroitin 4 and/or 6 sulfate, and a small amount of dermatan sulfate. An equal volume of control urine treated in a similar manner revealed no spots. The urinary GAGs of the affected dog were not detected after digestion with chondroitinase $\mathrm{ABC}$ (Fig. 3).

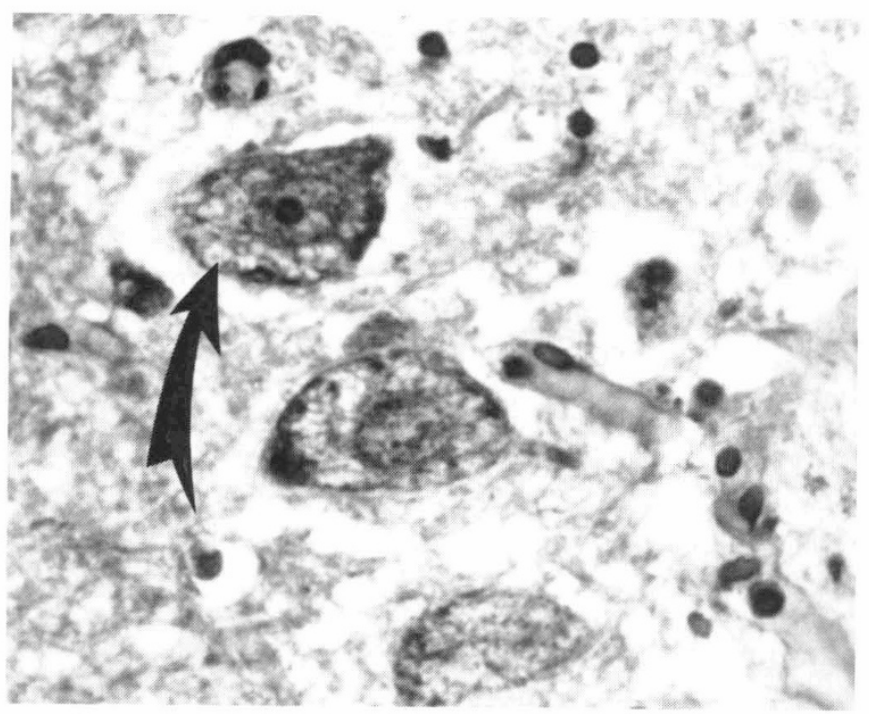

Fig. 4. A light micrograph of central nervous system neurons from the affected dog illustrating vacuolated cytoplasm. Cresyl violet, $\times 160$.
Because digestion with chondroitinase $\mathrm{AC}$ is complete under the conditions used, the GAG remaining was interpreted to be dermatan sulfate which migrated further than the standard, possibly because of a lowered molecular weight.

Pathology. The animal died suddenly from gastric dilatation at 14 months of age. Ascites, hydrothorax, and pulmonary congestion were present. There was generalized hepatomegaly, thickening of the atrioventricular heart valves, and generalized polyarthropathy with villous proliferation of synovial membranes in the joints of the appendicular skeleton. The lateral cerebral ventricles were moderately dilated.

Table 1. Lysosomal enzyme activities in liver from affected dog and control dogs*

\begin{tabular}{lcccc}
\hline & & \multicolumn{3}{c}{ Controls } \\
\cline { 3 - 5 } \multicolumn{1}{c}{ Enzyme } & Affected & Mean & Range $(n)$ \\
\hline$\beta$-Glucuronidase & 4.8 & 403 & $253-545$ & $(7)$ \\
$\beta$-Hexosaminidase A & 1050 & 2290 & $1010-3630$ & $(6)$ \\
$\beta$-Hexosaminidase B & 2130 & 2180 & $1015-3490$ & $(6)$ \\
$\alpha$-Hexosaminidase $\dagger$ & 39.6 & 39.8 & $32.9-46.5$ & $(5)$ \\
$\alpha$-L-Iduronidase $\dagger$ & 390 & 409 & $373-465$ & $(3)$ \\
$\alpha$-Galactosidase A & 34.9 & 37.9 & $32.2-39.1$ & $(3)$ \\
$\beta$-Galactosidase & 88.7 & 123 & $89-214$ & $(6)$ \\
Arylsulfatase A & 20.1 & 26.1 & $16.2-35.7$ & $(5)$ \\
Arylsulfatase B & 13.9 & 17.3 & $11.0-24.0$ & $(5)$ \\
Acid $\alpha$-mannosidase & 487 & 367 & $250-627$ & $(5)$ \\
Acid $\beta$-mannosidase & 386 & 500 & $265-851$ & $(3)$ \\
$N$-Acetyl-D-galactosamine-6- & & & & \\
$\quad$ sulfate sulfatase & & & & \\
$\quad \%$ Substrate released & 21.3 & 14.5 & $13.2-15.8$ & $(2)$ \\
$\quad$ Units/mg protein & 16.2 & 5.6 & $5.5-5.7$ & $(2)$ \\
\hline
\end{tabular}

${ }^{*}$ Activity is expressed as $\mathrm{nmol} / \mathrm{h} / \mathrm{mg}$ of protein; $\dagger, \mathrm{pmol} / \mathrm{h} / \mathrm{mg}$ of protein.

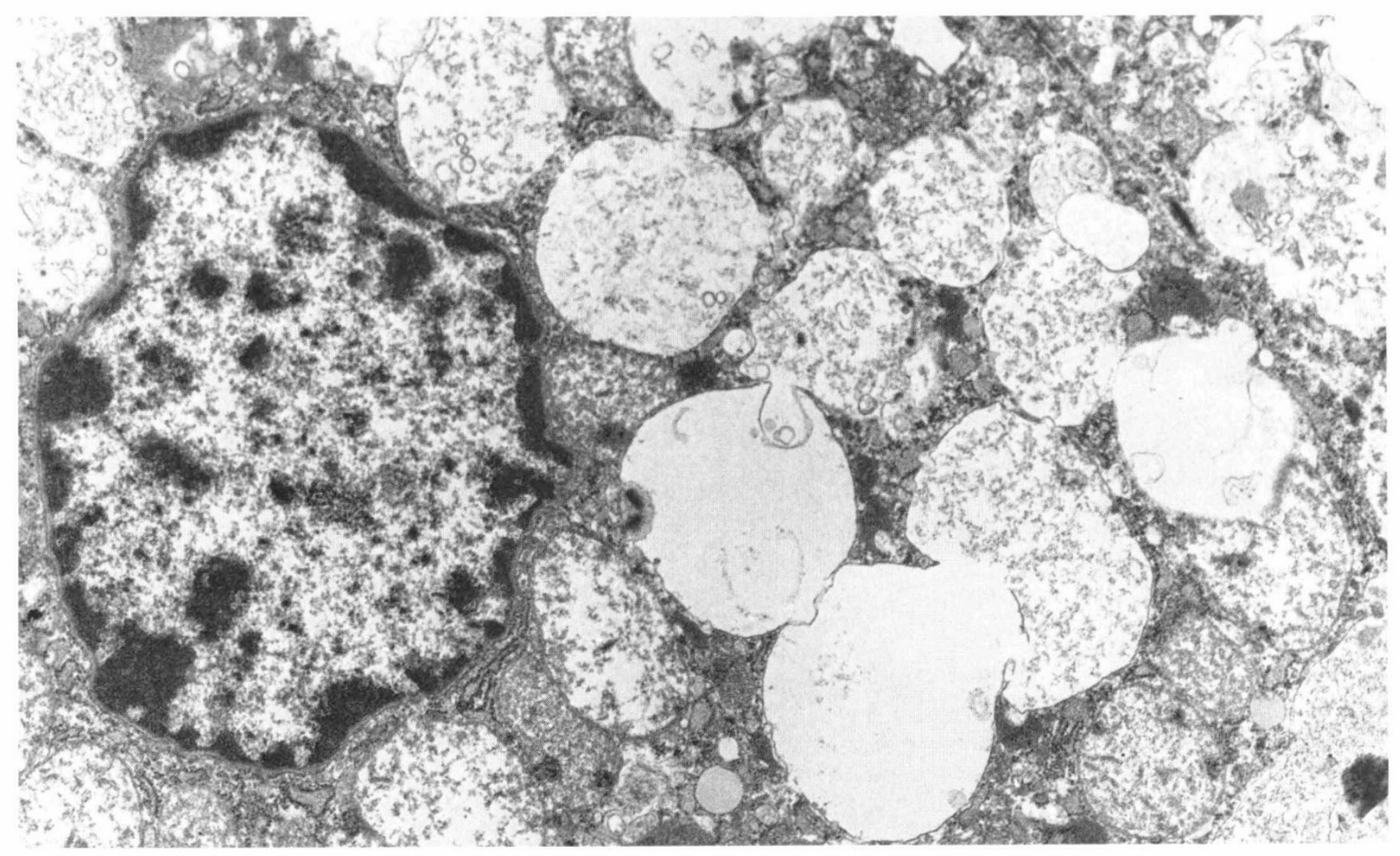

Fig. 5. An electron micrograph of an hepatocyte illustrating membrane-bound vacuoles which contain granular material. Lead citrate-uranyl acetate, $\times 4800$. 
Table 2. $\beta$-D-Glucuronidase activity in liver expressed as $\mathrm{nmol} / \mathrm{h} / \mathrm{mg}$ of protein*

\begin{tabular}{|c|c|c|c|c|}
\hline & \multicolumn{4}{|c|}{ Substrates } \\
\hline & \multicolumn{2}{|c|}{$\begin{array}{l}\text { 4-Methylumbelli- } \\
\text { feryl glucuronide }\end{array}$} & \multicolumn{2}{|c|}{$\begin{array}{l}\text { Nitrophenol } \\
\text { glucuronide }\end{array}$} \\
\hline & Mean & Range (n) & Mean & Range $(n)$ \\
\hline Affected & 4.8 & $0.17-7.9$ (3) & 49 & $11-89 \quad(5)$ \\
\hline Sire & 224 & $222-226(2)$ & 223 & $157-256(4)$ \\
\hline Dam & 59 & $51-67$ & 117 & $97-137(5)$ \\
\hline Half-sibling & & & 156 & (1) \\
\hline Control & 344 & $184-545(11)$ & 286 & $140-422(4)$ \\
\hline
\end{tabular}

${ }^{*} n$ indicates duplicate preparations for affected, sire, dam, and halfsibling; the number of control animals.

By light microscopy, vacuolated cytoplasm was observed in hepatocytes, keratocytes, fibroblasts, chondrocytes and cells of the synovial membrane, retinal pigment epithelium, and cardiac valves. Neurons with cytoplasmic vacuoles (Fig. 4) were clearly demonstrated in the spinal cord, hippocampal ganglion cells, pyramidal cells, sensory VI nucleus, and cerebral cortex. Evidence of storage was not definitive in other areas of the brain due to relatively poor fixation. Electron microscopy demonstrated membrane-bound cytoplasmic inclusions in polymorphonuclear leukocytes, hepatocytes (Fig. 5), keratocytes and cells of the conjunctiva, retinal pigment epithelium, synovium, heart valves, and spleen.

Enzyme assays. The activities of 12 lysosomal hydrolases were determined in liver from the affected and control dogs (Table 1). The activity of $\beta$-glucuronidase was reduced to less than $2 \%$ of the normal mean value in normal controls. Liver obtained by biopsy from the sire and dam and postmortem liver from a halfsibling were assayed for $\beta$-glucuronidase activity (Table 2) which revealed half-normal and less than half-normal activity in the half-sibling and dam, respectively, but near normal activity in the sire.

Genetics. The affected dog was the product of a consanguineous mating (father-daughter) (Fig. 1). Two siblings from a previous litter by the same parents were described as having a phenotype similar to the propositus. The historical data combined with the liver enzyme data of the half-sibling are consistent with an autosomal recessive pattern of inheritance as is seen in the human disease.

\section{DISCUSSION}

Since the first description of a human patient with $\beta$-glucuronidase deficiency by Sly et al. (19) in 1973, 15 additional cases have been reported in man (summarized in Ref. 9). Phenotypic variation has been observed among these patients ranging from severe mental retardation with skeletal abnormalities, corneal clouding, and hepatosplenomegaly to normal intelligence and stature with little or no corneal clouding or skeletal abnormality. The clinical phenotype of the affected dog most closely resembled that of the more severely affected human patients.

The detection of heterozygotes by the determination of intermediate levels of $\beta$-glucuronidase activity in parents of affected children has not been uniformly successful. Eleven obligate heterozygotes (parents of affected offspring) had levels of $\beta$ glucuronidase activity in cultured fibroblasts, serum, or white blood cells that overlapped the normal range, similar to that observed for the sire of the affected dog. At present, there is no adequate explanation for this observation.

Postmortem studies in human MPS VII patients have been limited $(9,22)$; antemortem studies of blood, bone marrow, cultured fibroblasts, and liver have been reported (6). The widely disseminated cytoplasmic vacuolation described in these patients was similar to that seen in the dog. The presence of storage within neurons is expected in human patients with lysosomal storage disease and mental retardation. Neuronal storage has been described in one human MPS VII patient (22). The affected dog had evidence of storage in central nervous system neurons without obvious clinical signs of neurologic disease, as has also been reported in cats and dogs with MPS I (7, 17). In animals, clinical signs which can be related to mental retardation are difficult to evaluate.

Previously, a deficiency of $\beta$-glucuronidase activity has been reported in the $\mathrm{C} 3 \mathrm{H}$ mouse strain (13). However, these mice have about $20 \%$ of the activity usually observed in mice and do not accumulate GAG, nor do they have morphologic or clinical manifestations of disease. This report describing canine MPS VII therefore represents the first animal model of $\beta$-glucuronidase deficiency with associated disease. The parents of the affected dog are being bred to produce a colony of affected animals which should provide an opportunity to investigate the pathogenesis of this disorder, as well as approaches to therapy.

Acknowledgments. The authors wish to thank Dr. J. T. McGrath and Dr. G. D. Aguirre for assistance with interpretation of neuro- and ophthalmic pathology, respectively, and J. Collenberg for technical assistance in electron microscopy.

\section{REFERENCES}

1. Berry HK, Spinanger J 1960 A paper spot test useful in study of Hurler's syndrome. J Lab Clin Med 55:136

2. Desnick RJ, Allen KY, Desnick SJ, Raman MK, Bernlohr RW, Krivit W 1973 Fabry disease: enzymatic diagnosis of hemizygotes and heterozygotes. Alphagalactosidase activities in plasma, serum and leukocytes. J Lab Clin Med $81: 157$

3. Desnick RJ, Sharp HL, Grabowski GA, Brunning RD, Sung JH, Quie PG, Ikonne JU 1976 Mannosidosis: clinical, ultrastructural, immunologic and biochemical studies. Pediatr Res 10:985

4. DiFerrante N, Neri G, Neri ME, Hogsett WE 1972 Measurement of urinary glycosaminoglycans with quaternary ammonium salts: an extension of the method. Connect Tissue Res 1:93

5. Fiddler MB, Desnick RJ 1977 Enzyme therapy. VII. Differential in vivo retention of bovine hepatic, renal and splenic $\beta$-glucuronidase and evidence for enzyme stabilization by intermolecular exchange. Arch Biochem Biophys 179:397

6. Gehler J, Canty M, Tolksdorf M, Spranger J 1974 Mucopolysaccharidosis. VII. $\beta$-Glucuronidase deficiency. Humangenetik 23:149

7. Haskins ME, Jezyk PF. Desnick RJ, McDonough SK, Patterson DF 1979 Alpha-L-iduronidase deficiency: a model of mucopolysaccharidosis I. Pediatr Res 13:1294

8. Ikonne JU, Rattazzi MC, Desnick RJ 1975 Characterization of Hex S. The major residual $\beta$-hexosaminidase activity in Sandhoff disease. Am J Hum Genet 27:639

9. Irani D, Kim H-S, El-Hibri H, Dutton RV, Beaudet A, Armstrong D 1983 Postmortem observations on $\beta$-glucuronidase deficiency presenting as hydrops fetalis. Ann Neurol 14:486

10. Jezyk PF, Haskins ME, Patterson DF, Mellman WJ, Greenstein M 1977 Mucopolysaccharidosis in a cat with arylsulfatase B deficiency: a model of Maroteaux-Lamy syndrome. Science 198:834

11. McGovern MM, Vine DT, Haskins ME, Desnick RJ 1981 An improved method for heterozygote identification in feline and human mucopolysaccharidosis. VI. Arylsulfatase B deficiency. Enzyme 26:206

12. McKusick VA. Neufeld EF 1983 The mucopolysaccharide storage diseases. In Stanbury JB, Wyngaarden JB, Fredrickson DS, Goldstein JL, Brown MS (eds) The Metabolic Basis of Inherited Disease, 5th ed. McGraw Hill, New York, pp 751-777

13. Morrow AG, Greenspan EM, Carroll DM 1949 Liver $\beta$-glucuronidase activity of inbred mouse strains. J Natl Cancer Inst 10:657

14. Mourão PAS, Donnelly PV, DiFerrante DT, DiFerrante N 1981 The pericellular glycosaminoglycans of human fibroblasts defective for low density lipoproteins receptors. Biochem Int 2:95

15. O'Brien JS 1972 Sanfilippo syndrome: profound deficiency of alpha-acetylglucosaminidase activity in organs and skin fibroblasts from Type-B patients. Proc Natl Acad Sci USA 69:1720

16. Shafit-Zagardo B, Devine EA, Desnick RJ 1980 Electrophoretic separation of neutral and acid $\beta$-glucosidase isozymes in normal tissues. Biochim Biophys Acta $614: 459$

17. Shull RM, Munger RJ, Spellacy E, Hall CW, Constantopoulos G, Neufeld EF 1982 Canine $\alpha$-L-iduronidase deficiency: a model of mucopolysaccharidosis Am J Pathol 109:244

18. Skoog WA, Beck WS 1956 Studies on the fibrinogen, dextran and phytohemagglutinin methods of isolating leukocytes. Blood 11:436

19. Sly WS, Quinton BA, McAlister WH, Rimoin DL 1973 Beta-glucuronidase deficiency. Report of clinical, radiologic and biochemical features of a new mucopolysaccharidosis. J Pediatr 82:249

20. Spurr AR 1969 A low-viscosity epoxy resin embedding medium for electron 
microscopy. J Ultrastruct Res 26:31

21. Toma S, DiFerrante DT, Tenni R, DiFerrante N 1981 Preparation from keratan sulfate of substrates for the measurement of 2-actamido-2-deoxy-Dglucose 6 -sulfate sulfatase and $(1 \rightarrow 3)-N$-acetyl- $\beta$-D-glucosaminidase. Car- bohydr Res 88:93

22. Wilson D, Melnike E, Sly W, Marksbery WR 1982 Neonatal beta-glucuronidase deficiency mucopolysaccharidosis (MPS VII). J Neuropathol Exp Neurol $41: 344$

\title{
Microvillus Membrane Differentiation: Quantitative Difference in Cholera Toxin Binding to the Intestinal Surface of Newborn and Adult Rabbits
}

\author{
J. L. BRESSON, K. Y. PANG, AND W. A. WALKER \\ Pediatric Gastrointestinal and Nutrition Unit, Massachusetts General Hospital and the Pediatric Department, \\ Harvard Medical School, Boston, Massachusetts, 02114
}

\begin{abstract}
Summary
Microvillus membranes (MVM) were isolated from newborn and adult New Zealand rabbit small intestine. The isolation procedure provided a mean enrichment of $25 \pm 4$ for sucrase activity in adult preparations and of $27 \pm 3$ for lactase activity in newborn preparations. These purified MVM were incubated with increasing concentrations of ${ }^{125} \mathrm{I}$-labeled cholera toxin (CT). ${ }^{125} \mathrm{I}$ CT binding to adult MVM reached saturation at $6.4 \times 10^{-9} \mathrm{M}$; in contrast ${ }^{125} \mathrm{I}-\mathrm{CT}$ binding to newborn MVM did not reach saturation but instead continued to increase with increasing ${ }^{125} \mathrm{I}-$ CT concentrations. Scatchard plot analysis of adult data supported the existence of a single binding site $\left(K_{d}=1.2 \pm 0.2 \times\right.$ $10^{-9} \mathrm{M}$ ); analysis of newborn data, however, suggested the existence of additional binding sites, as ${ }^{125} \mathrm{I}-\mathrm{CT}$ binding to newborn MVM was inhibited by preincubation with unlabeled CT. These results show that $\mathrm{CT}$ binding to both preparations is quantitatively different and is higher in newborn preparations. This difference may be accounted for by the existence of additional binding sites in newborn MVM preparations in contrast to the presence of only the unique receptor previously reported in adult MVM preparations.
\end{abstract}

\section{Abbreviations}

MVM, microvillus membrane

CT, cholera toxin

Hepes, 4-(2-hydroxyethyl)-1-piperazineethanesulfonic acid

In many species, the small intestines undergo important modifications during postnatal development such as structural changes of the epithelial layer (6) or changes in MVM enzymatic activities (25). These modifications are likely to induce, as a

\footnotetext{
Received March 9, 1983; accepted January 9, 1984.
}

Reprints requests may be addressed to J. L. Bresson, Service de Gastroenterologie Pediatrique et de Nutrition, Hopital des Enfants Malades, 149, Rue de Sevres, 75730 Paris Cedex 15, France.

This work was supported by Grants GM21700, HD12437, and AM16269, from the National Institute of Health. K. Y. P. is a Trainee in Gastrointestinal Research (T32-AM07191). result, changes in MVM surface properties that may be reflected by events such as the "closure" of macromolecular transport through the small intestine (24). Comparison of MVM surface properties between newborn and adult are not yet available. Indirect approaches, such as those of Extler and Branstrator (9), have been used to study the lectin-binding capacity of small intestine mucosa during postnatal development; however, examination of histologic preparations under light microscopic conditions does not allow for a more precise conclusion about MVM-binding properties to be made.

In order to study MVM surface property changes with differentiation, we designed an in vitro system using MVM vesicles purified from newborn and adult rabbit small intestine, and tested these vesicles for binding characteristics using a molecular probe with an extensively studied microvillus receptor.

\section{MATERIALS AND METHODS}

Microvillus membrane isolation was performed according to a modification (3) of the $\mathrm{Ca}^{2+}$ precipitation method (27). Fasted adult ( $n=8$; more than 8 weeks old) and newborn $(n=50 ; 12$ to $24 \mathrm{~h}$ of life) New Zealand rabbits (Margaret's Home Farm, Greenfield, MA) were sacrificed and the small intestines were removed and washed with cold saline. The following steps were carried out at $4^{\circ} \mathrm{C}$. Scrapings of the whole adult mucosa or 4-5$\mathrm{cm}$ pieces of opened newborn small intestines were homogenized in $300 \mathrm{mM}$ mannitol, $12 \mathrm{mM}$ Tris buffer at $\mathrm{pH} 7.4$, diluted 1:6, and filtered on a $40-\mu \mathrm{m}$ nylon mesh (Tetko, Elmsford, NY). $\mathrm{CaCl}_{2}$ was added to a final concentration of $10 \mathrm{mM}$. After 15 $\min$ on ice, homogenates were centrifuged at $2500 \times g$ for 15 min on a Beckman J21B centrifuge with a JS7.5 rotor. Supernatants were saved and centrifuged with a JA20 rotor at 28,000 $\times g$ for $30 \mathrm{~min}$, then suspended in $50 \mathrm{mM}$ mannitol, $1 \mathrm{mM}$ Hepes buffer at $\mathrm{pH} 7.4$, and centrifuged again. The pellets (crude preparation) were resuspended in $1 \mathrm{mM}$ Hepes buffer and applied on the top of 10 to $60 \%$ sucrose gradients (Sigma Co., St. Louis, MO) which were centrifuged at $64,000 \times g$ for $25 \mathrm{~min}$ on a Beckman L5-65 ultracentrifuge with a SW 28 rotor. Bands were removed from the top, diluted $1: 10$, centrifuged at $100,000 \times g$ for $60 \mathrm{~min}$ on a $50 \mathrm{Ti}$ rotor. Pellets (final preparation) were 